\title{
Bulk elastic waves with unidirectional backscattering-immune topological states in a time-dependent superlattice
}

Cite as: J. Appl. Phys. 118, 063103 (2015); https://doi.org/10.1063/1.4928619

Submitted: 08 May 2015. Accepted: 03 August 2015. Published Online: 14 August 2015

N. Swinteck, S. Matsuo, K. Runge, J. O. Vasseur, P. Lucas, and P. A. Deymier
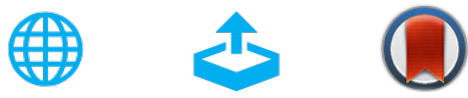

View Online

Export Citation

CrossMark

\section{ARTICLES YOU MAY BE INTERESTED IN}

Helical edge states and topological phase transitions in phononic systems using bi-layered lattices

Journal of Applied Physics 119, 084305 (2016); https://doi.org/10.1063/1.4942357

Reconfigurable topological insulator for elastic waves

The Journal of the Acoustical Society of America 146, 773 (2019); https://

doi.org/10.1121/1.5114920

Brillouin scattering-like effect and non-reciprocal propagation of elastic waves due to spatiotemporal modulation of electrical boundary conditions in piezoelectric media

Applied Physics Letters 110, 061901 (2017); https://doi.org/10.1063/1.4975680

Challenge us.

What are your needs for periodic signal detection?

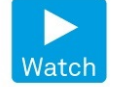

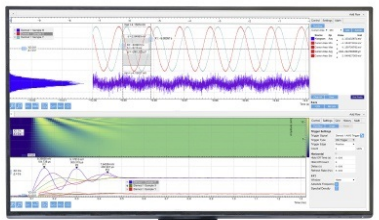

0
Zurich

- Instruments 


\title{
Bulk elastic waves with unidirectional backscattering-immune topological states in a time-dependent superlattice
}

\author{
N. Swinteck, ${ }^{1, a)}$ S. Matsuo, ${ }^{1}$ K. Runge,${ }^{1}$ J. O. Vasseur, ${ }^{2}$ P. Lucas, ${ }^{1}$ and P. A. Deymier ${ }^{1}$ \\ ${ }^{1}$ Department of Materials Science and Engineering, University of Arizona, Tucson, Arizona 85721, USA \\ ${ }^{2}$ Institut d'Electronique, de Micro-électronique et de Nanotechnologie, UMR CNRS 8520, Cité Scientifique, \\ 59652 Villeneuve d'Ascq Cedex, France
}

(Received 8 May 2015; accepted 3 August 2015; published online 14 August 2015)

\begin{abstract}
Recent progress in electronic and electromagnetic topological insulators has led to the demonstration of one way propagation of electron and photon edge states and the possibility of immunity to backscattering by edge defects. Unfortunately, such topologically protected propagation of waves in the bulk of a material has not been observed. We show, in the case of sound/elastic waves, that bulk waves with unidirectional backscattering-immune topological states can be observed in a time-dependent elastic superlattice. The superlattice is realized via spatial and temporal modulation of the stiffness of an elastic material. Bulk elastic waves in this superlattice are supported by a manifold in momentum space with the topology of a single twist Möbius strip. Our results demonstrate the possibility of attaining one way transport and immunity to scattering of bulk elastic waves. (C) 2015 AIP Publishing LLC. [http://dx.doi.org/10.1063/1.4928619]
\end{abstract}

\section{INTRODUCTION}

Topological electronic ${ }^{1}$ or electromagnetic ${ }^{2-4}$ insulators have the astonishing property of unidirectional, backscattering-immune edge states. This property is associated with the non-conventional topology of the wave states. So far, the observation of topologically protected propagation of electronic or electromagnetic waves has been limited to the edges of the material. In one-dimensional (1D) topological insulators, topologically protected edge states are zero-dimensional (OD) and cannot exhibit transport. In twodimensional (2D) and three-dimensional (3D) topological insulators, the edge states are $1 \mathrm{D}$ and $2 \mathrm{D}$, respectively, and can lead to topologically protected unidirectional propagation along the materials' edges or surfaces. The highly desirable property of non-reciprocal and topologically protected propagation of waves (electronic, electromagnetic, or any other type of wave such as sound or elastic waves) inside the bulk of a material has not yet been demonstrated. Substantial effort has been directed toward proposing and demonstrating non-reciprocal acoustic materials that rely on non-linear elastic materials, ${ }^{5-8}$ non-linear magneto-elastic media, ${ }^{9}$ or resonators containing moving fluids. ${ }^{10}$ These approaches of breaking reciprocity, however, do not offer topological immunity to scattering.

Non-reciprocal topological frequency bands have been shown to emerge in finite slabs (infinitely periodic in 1D) formed out of 2D electromagnetic lattices of metamaterial components $^{11}$ and 2D magneto-electric photonic crystals. ${ }^{12}$ In these systems at distinct frequencies, partial nonreciprocity may arise when the corresponding forward and backward-propagating wavevectors do not have the same magnitude. Spatio-temporal modulation of the properties of materials has also been used to achieve one-way wave

\footnotetext{
a) Author to whom correspondence should be addressed. Electronic mail: swinteck@email.arizona.edu.
}

propagation. Dynamically modulated photonic structures can transmit light in a single direction. ${ }^{13,14}$ This approach is based on subjecting the photonic structure to a spatial and temporal modulation of the refractive index that results in direction-dependent frequency and momentum shifts leading to one way propagation of light. The interaction between photons and the modulation is interpreted in terms of inter-band transitions in the space of the time-independent wave functions of the photonic structure. ${ }^{15}$ These timeindependent wave functions exhibit the conventional topology imparted by the photonic structure and the transitions are constrained by the usual selection rules. Furthermore, time- and space-variant phononic systems have been shown to enable control of phonon dispersion in the frequency and wavenumber domains. ${ }^{16-18}$

Here, we demonstrate unidirectional propagation of sound/elastic waves in a 1D time-dependent superlattice by breaking the symmetry of their dispersion behavior. In contrast to the transition interpretation, the time-dependence of the interaction is included in the elastic wave function itself leading to a more comprehensive non-conventional topological interpretation of the states and of their topological constraints. Moreover, in contrast to edge states, we report unidirectional propagation of bulk elastic waves that can also lead to immunity to backscattering by defects in the bulk of a material.

\section{Time-dependent elastic superlattice}

We consider the periodic spatial modulation of the stiffness of a 1D elastic medium and its directed temporal evolution that breaks time reversal symmetry. The bulk elastic states of this time-dependent superlattice do not possess the conventional mirror symmetry in momentum space leading to non-reciprocity in the direction of propagation of the waves. The wave functions of bulk elastic waves are supported by a 
manifold in momentum space that has the non-conventional torsional topology of a Möbius strip with a single twist.

To realize the time-dependent elastic superlattice, we consider the propagation of longitudinal elastic waves along a 1D material supporting a spatial and temporal sinusoidal modulation of its stiffness. Unique properties of some materials such as the giant photo-elastic effects in chalcogenide glasses ${ }^{19}$ can be exploited to practically achieve the desired stiffness modulations by, for instance, illuminating the material with light of spatially and temporally varying intensity. It has been shown that illuminating Ge-Se chalcogenide glasses with near bandgap laser radiation of increasing power results in a reduction of the longitudinal elastic constant $\left(C_{11}\right)$ by nearly $50 \%$. This photo-softening is athermal and reversible making it ideal as a means to realize timedependent modulations. The stiffness modulation may also be achieved by various other means such as the application of time-space dependent magnetic fields to a magneto-elastic medium, or the modulation of voltage applied to a medium composed of piezoelectric elements, or the mechanical stimulation of a non-linear elastic medium. Here, we consider the medium to be composed of a Ge-Se chalcogenide glass of composition $\mathrm{GeSe}_{4}{ }^{19}$ Depending on the power of the laser irradiating the glass, $C_{11}$ values for $\mathrm{GeSe}_{4}$ can vary between 9.2 $\mathrm{GPa}$ (full-power) and $18.4 \mathrm{GPa}$ (zero-power). ${ }^{19} \mathrm{We}$ assume constant density for $\mathrm{GeSe}_{4}\left(4361 \mathrm{~kg} / \mathrm{m}^{3}\right)$, therefore the minimum and maximum values of $C_{11}$ coincide with sound velocities of $1452 \mathrm{~m} / \mathrm{s}$ and $2054 \mathrm{~m} / \mathrm{s}$, respectively. By itself, a block of $\mathrm{GeSe}_{4}$ is merely a homogeneous medium with constant elastic properties. If, however, the glass was placed under an array of lasers, the elastic properties of the material could be modulated in space and/or time by dynamically adjusting the power of each element in the laser array. This configuration is the basis for the time-dependent elastic superlattice described hereafter.

The vibrational properties of this system are investigated numerically. We represent the time-dependent elastic superlattice by a discrete 1D mass-spring system with a spatial sinusoidal modulation of the stiffness of the springs that propagates in time with the velocity $\pm V$ (Fig. 1(a)). Individual masses $\left(m=4.361 \times 10^{-9} \mathrm{~kg}\right)$ are equally spaced by $a=0.1 \mathrm{~mm}$. The masses are connected by springs that can vary in stiffness between 920000 and $1840000 \mathrm{~kg} \cdot \mathrm{s}^{-2}$. The study of the dynamics of the discretized time-dependent model superlattice is amenable to the method of molecular dynamics (MD). For the calculation of the elastic band structure of the superlattice, we use a $1 \mathrm{D}$ chain that contains $N=3200$ masses with Born-Von Karman boundary conditions. The system takes the form of a ring. We have chosen the value of 100 inter-mass spacings for the period of the stiffness modulation, $L$. The dynamical trajectories generated by the MD simulation are analyzed within the framework of the Spectral Energy Density (SED) method $^{20}$ for generating the elastic band structure of the model superlattice. To ensure adequate sampling of the system's phase-space, our reported SED calculations represent an average over 15 individual MD simulations each with time step of $1.5 \mathrm{~ns}$ and total simulation time of $2^{22}$ time steps. We report in Figures 1(b) and $1(\mathrm{c})$ the calculated band structure of the superlattice for two velocities of the spatial modulation, namely, 0 and $350 \mathrm{~m} / \mathrm{s}$.

The band structure of the time-independent superlattice (Fig. 1(b)) exhibits the usual band folding features with gaps forming at the edge of the Brillouin zone. The band structure has the mirror symmetry in momentum space about the origin characteristic of time reversal symmetry. In this case, since the system studied takes the form of a ring, it supports degenerate counter-propagating elastic Eigenmodes. One consequence of the time-dependence of the stiffness modulation is the loss of the mirror symmetry in $k$-space which is indicative of breaking time reversal symmetry (Fig. 1(c)). In addition to the presence of bands reminiscent of the timeindependent bands, the band structure of the time-dependent superlattice contains a series of faint frequency shifted bands. The frequency shift amounts to multiples of $\Omega=\frac{2 \pi V}{L}$. The intensity of these bands decreases as the shift in frequency increases. More remarkable is the formation of hybridization gaps between the frequency-shifted bands and the original time-independent bands. Two such gaps appear in the positive-frequency, positive-wavenumber quadrant of the first Brillouin zone at the same wavenumber $+k_{g}$. Such gaps do not appear in the positive-frequency, negative-wavenumber quadrant (i.e., at $-k_{g}$ ) thus indicating the loss of mirror symmetry. Changing the sign of the modulation velocity leads to a horizontal flip of the band structure. In the frequency range corresponding to the band gaps, the timedependent ring-like superlattice does not support degenerate counter-propagating elastic Eigenmodes anymore. At these frequencies, the degeneracy in the direction of propagation is lifted and the time-dependent mass-spring ring supports lefthanded or right-handed modes depending upon the velocity of the modulation.

\section{RESULTS AND DISCUSSION}

\section{Multiple time scales perturbation theory of time-dependent superlattice}

To illustrate the origin of the loss of mirror symmetry in the band structure of the time-dependent superlattice, we construct perturbative solutions to the elastic wave functions. In the long-wavelength limit, propagation of longitudinal elastic waves in a one-dimensional medium perturbed by a spatio-temporal modulation of its stiffness, $C(x, t)$, obeys the following equation of motion:

$$
\rho \frac{\partial^{2} u(x, t)}{\partial t^{2}}=\frac{\partial}{\partial x}\left(C(x, t) \frac{\partial u(x, t)}{\partial x}\right) .
$$

In Equation (1), $u(x, t)$ is the displacement field and $\rho$ is the mass density of the medium. For the sake of analytical simplicity, we choose a sinusoidal variation of the stiffness with position and time

$$
C(x, t)=C_{0}+2 C_{1} \sin (K x+\Omega t),
$$

where $C_{0}$ and $C_{1}$ are positive constants. $K=\frac{2 \pi}{L}$, where $L$ is the period of the stiffness modulation. $\Omega$ is a frequency associated with the velocity of the stiffness modulation, $V$. The 
(a)
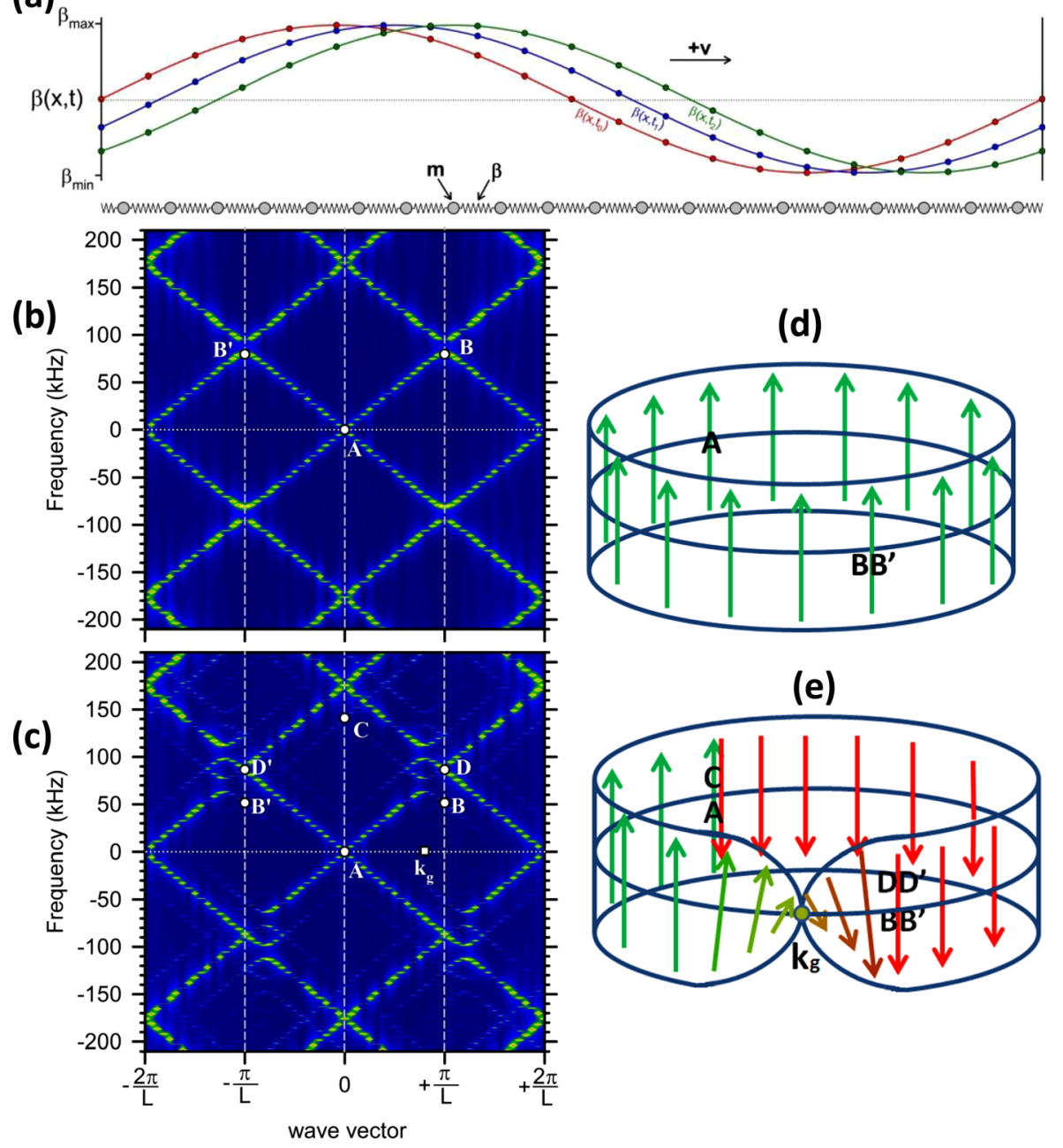

(d)

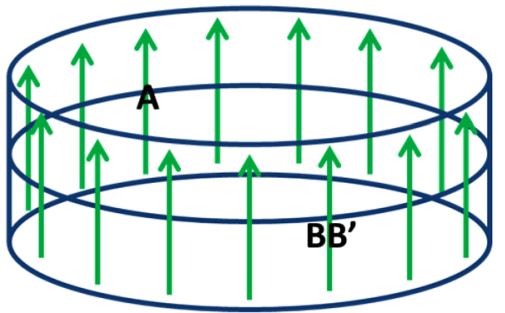

(e)

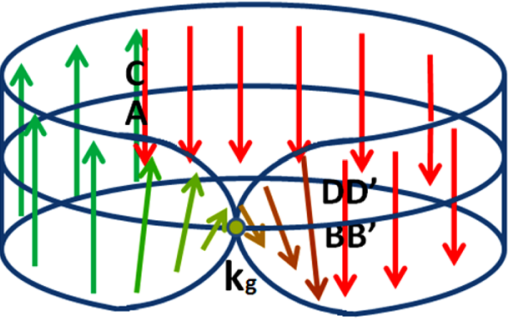

FIG. 1. Elastic waves in time-dependent elastic superlattice: (a) One-dimensional harmonic mass-spring system with spatial and temporal sinusoidal modulation of the spring stiffness: $\beta(x, t)$ as a realization of an elastic time-dependent superlattice. The spatial modulation propagates in time with the velocity $\pm \boldsymbol{V}$. (b) and (c) Calculated elastic wave band structure in the cases of modulation velocities of 0 (time-independent superlattice) and $350 \mathrm{~m} / \mathrm{s}$, respectively. (d) Illustration of the conventional momentum space ( $k$-space) manifold supporting Bloch waves in the time-independent superlattice. Parallel transport of a vector field along a $\frac{2 \pi}{L}$ closed path in $k$ space that starts and finishes at the origin of the band structure A and goes through points B and $\mathrm{B}^{\prime}$ shows no accumulation of phase as the tangent vector does not change orientation along the path. (e) Illustration of the $k$-space manifold supporting elastic waves in the time-dependent superlattice. The manifold takes the form of a Möbius strip with a single twist centered on the wavenumber $k_{g}$ corresponding to the band gaps in the band structure. The accumulation of phase along a $\frac{4 \pi}{L}$ long closed path in $k$ space that starts and ends at the origin A and goes through points $\mathrm{B}$, $\mathrm{B}^{\prime}, \mathrm{C}, \mathrm{D}$, and $\mathrm{D}^{\prime}$ is shown through parallel transport of a vector field. The amplitude of the wave function accumulates a $\pi$ phase every time the path crosses $k_{g}$. The phase change of the amplitude is represented by the change in orientation of the vector tangent to the manifold as it is transported along the closed path.

quantities $K$ and $V$ are independent. The sign of $\Omega$ determines the direction of propagation of the modulation. In this representation, the maximum stiffness of the chalcogenide material is $C_{11}^{\max }=C_{0}+2 C_{1}$.

The periodicity of the modulated one-dimensional medium suggests that we should be seeking solutions of Equation (1) in the form of Bloch waves

$$
u(x, t)=\sum_{k} \sum_{g} u(k, g, t) e^{i(k+g) x}
$$

where $x \in[0, L]$. The wave number $k$ is limited to the first Brillouin zone: $\left[\frac{-\pi}{L}, \frac{\pi}{L}\right]$ and $g=\frac{2 \pi}{L} m$ with $m$ being a positive or negative integer. With this choice of form for the solution and inserting Equation (2) into Equation (1), the equation of propagation takes the form

$$
\begin{aligned}
& \frac{\partial^{2} u(k+g, t)}{\partial t^{2}}+v_{a}^{2}(k+g)^{2} u(k+g, t) \\
& =i \varepsilon\left\{f\left(k^{\prime}\right) u\left(k^{\prime}, t\right) e^{i \Omega \mathrm{t}}+h\left(k^{\prime \prime}\right) u\left(k^{\prime \prime}, t\right) e^{-i \Omega \mathrm{t}}\right\},
\end{aligned}
$$

where $f(k)=K k+k^{2}, h(k)=K k-k^{2}, k^{\prime}=k+g-K$, and $k^{\prime \prime}=k+g+K$. In this equation, we have defined: $v_{a}^{2}=\frac{C_{0}}{\rho}$ and $\varepsilon=\frac{C_{1}}{\rho}$. We solve this equation by using the multiple-time scales perturbation method. ${ }^{21}$ For the sake of analytical simplicity, we treat $\varepsilon$ as a perturbation and write the displacement as a second order power series in the perturbation, namely,

$$
\begin{aligned}
u(k+ & \left.g, \tau_{0}, \tau_{1}, \tau_{2}\right) \\
= & u_{0}\left(k+g, \tau_{0}, \tau_{1}, \tau_{2}\right)+\varepsilon u_{1}\left(k+g, \tau_{0}, \tau_{1}, \tau_{2}\right) \\
& +\varepsilon^{2} u_{2}\left(k+g, \tau_{0}, \tau_{1}, \tau_{2}\right) .
\end{aligned}
$$


In Equation (5), $u_{i}$ with $i=0,1,2$ are displacement functions expressed to zeroth-order, first-order, and second-order in the perturbation. We have also replaced the single time variable, $t$, by three variables representing different time scales: $\tau_{0}=t, \tau_{1}=\varepsilon t$, and $\tau_{2}=\varepsilon^{2} t=\varepsilon^{2} \tau_{0}$. We can subsequently decompose Equation (4) into three equations: one equation to zeroth-order in $\varepsilon$, one equation to first-order in $\varepsilon$, and a third equation to second-order in $\varepsilon$. The zeroth-order equation represents propagation of an elastic wave in a homogeneous medium. Its solution is taking the form of the Bloch wave

$$
u_{0}\left(k+g, \tau_{0}, \tau_{1}, \tau_{2}\right)=a_{0}\left(k+g, \tau_{1}, \tau_{2}\right) e^{i \omega_{0}(k+g) \tau_{0}} .
$$

To zeroth-order, the dispersion relation takes the usual form: $\omega_{0}(k+g)=v_{a}|k+g|$.
The first order equation is used to solve for $u_{1}$

$$
\begin{aligned}
& \frac{\partial^{2} u_{1}\left(k+g, \tau_{0}, \tau_{1}, \tau_{2}\right)}{\partial \tau_{0}^{2}}+\omega_{0}^{2}(k+g) u_{1}\left(k+g, \tau_{0}, \tau_{1}, \tau_{2}\right) \\
& \quad+2 \frac{\partial^{2} u_{0}\left(k+g, \tau_{0}, \tau_{1}, \tau_{2}\right)}{\partial \tau_{1} \partial \tau_{0}} \\
& =i\left\{f\left(k^{\prime}\right) u_{0}\left(k^{\prime}, \tau_{0}, \tau_{1}, \tau_{2}\right) e^{i \Omega \tau_{0}}+h\left(k^{\prime \prime}\right) u_{0}\left(k^{\prime \prime}, \tau_{0}, \tau_{1}, \tau_{2}\right) e^{-i \Omega \tau_{0}}\right\} .
\end{aligned}
$$

The third term in Equation (7) is a secular term that is set to zero by assuming that the displacement, $u_{0}\left(k+g, \tau_{0}, \tau_{2}\right)$, is not a function of $\tau_{1}$. Subsequently, we will assume that the displacement at all orders of expansion is independent of odd time scales. The solution to Equation (7) is obtained in the form of the sum of homogeneous and particular solutions with split frequency

$$
\begin{aligned}
u_{1}\left(k+g, \tau_{0}, \tau_{2}\right)= & a_{1}\left(k+g, \tau_{2}\right) e^{i \omega_{0}(k+g) \tau_{0}}+i \frac{f\left(k^{\prime}\right) a_{0}\left(k^{\prime}, \tau_{2}\right)}{\omega_{0}^{2}(k+g)-\left(\omega_{0}\left(k^{\prime}\right)+\Omega\right)^{2}+i \varphi} e^{i\left(\omega_{0}\left(k^{\prime}\right)+\Omega\right) \tau_{0}} \\
& +i \frac{h\left(k^{\prime \prime}\right) a_{0}\left(k^{\prime \prime}, \tau_{2}\right)}{\omega_{0}^{2}(k+g)-\left(\omega_{0}\left(k^{\prime \prime}\right)-\Omega\right)^{2}+i \varphi} e^{i\left(\omega_{0}\left(k^{\prime \prime}\right)-\Omega\right) \tau_{0}} .
\end{aligned}
$$

We have introduced in the first-order solution given by Eq. (8) a small damping term $i \varphi$ to address the divergence of the two resonances that occur at $\omega_{0}^{2}(k+g)=$ $\left(\omega_{0}\left(k^{\prime}\right)+\Omega\right)^{2}$ and $\omega_{0}^{2}(k+g)=\left(\omega_{0}\left(k^{\prime \prime}\right)-\Omega\right)^{2}$. We will later take the limit $\varphi \rightarrow 0$. The first term in the righthand-side of Equation (8) is the solution of the homogeneous part of Equation (7) and takes the same form as the zeroth-order solution of Equation (6). The other two terms are particular solutions. They are equivalent to solutions for a driven harmonic oscillator. As seen in Figure 1(c), the particular solutions introduce additional dispersion curves in the band structure of the time-dependent superlattice obtained by shifting the zeroth-order band structure by $\pm \Omega$. The faint intensity of these bands reflects the non-resonant conditions for the amplitudes in Eq. (8). We also make the important observation that there is a phase difference of $\pi$ between the first-order particular solution and the homogeneous (and zerothorder) solutions of Equation (8). This phase is due to the change in sign of the amplitude of the first-order displacement function as the wave number is varied across the resonance. Finally, the second order equation of motion is given by

$$
\begin{aligned}
& \frac{\partial^{2} u_{2}\left(k+g, \tau_{0}, \tau_{2}\right)}{\partial \tau_{0}^{2}}+\omega_{0}^{2}(k+g) u_{2}\left(k+g, \tau_{0}, \tau_{2}\right) \\
& \quad+2 \frac{\partial^{2} u_{0}\left(k+g, \tau_{0}, \tau_{2}\right)}{\partial \tau_{2} \partial \tau_{0}} \\
& =i\left\{f\left(k^{\prime}\right) u_{1}\left(k^{\prime}, \tau_{0}, \tau_{2}\right) e^{i \Omega \tau_{0}}+h\left(k^{\prime \prime}\right) u_{1}\left(k^{\prime \prime}, \tau_{0}, \tau_{2}\right) e^{-i \Omega \tau_{0}}\right\} .
\end{aligned}
$$

Inserting Equation (8) into (9) leads to terms of the form $e^{i \omega_{0}(k+g) \tau_{0}}$ in the right-hand-side of the equation. These terms lead to secular behavior that can be cancelled by equating them to the third term in the left-hand-side of the equation. Introducing, $a_{0}\left(k+g, \tau_{2}\right)=\alpha_{0}(k+g) e^{i \gamma \tau_{2}}$, one may rewrite $u_{0}\left(k+g, \tau_{0}, \tau_{2}\right) \quad$ as $\quad u_{0}\left(k+g, \tau_{0}, \tau_{2}\right)=\alpha_{0}(k+g) e^{i \gamma \tau_{2}} e^{i \omega_{0}(k+g) \tau_{0}}$ $=\alpha_{0}(k+g) e^{i\left[\omega_{0}(k+g)+\gamma \varepsilon^{2}\right] \tau_{0}}=\alpha_{0}(k+g) e^{i \omega_{0}^{*}(k+g) \tau_{0}}$. Then, one obtains a correction to $\omega_{0}(k+g)$, leading to a frequency shift and damping. This frequency shift is most pronounced for values of the wave number leading to strong resonances in Equation (8) and is given by

$$
\delta \omega_{0}(k+g)=\omega_{0}^{*}(k+g)-\omega_{0}(k+g)=\varepsilon^{2}(\gamma)_{p p}=\frac{\varepsilon^{2}}{2 \omega_{0}(k+g)}\left\{\begin{array}{c}
f\left(k^{\prime}\right) h(k+g)\left(\frac{1}{\omega_{0}^{2}\left(k^{\prime}\right)-\left(\omega_{0}(k+g)-\Omega\right)^{2}}\right)_{p p} \\
+h\left(k^{\prime \prime}\right) f(k+g)\left(\frac{1}{\omega_{0}^{2}\left(k^{\prime \prime}\right)-\left(\omega_{0}(k+g)+\Omega\right)^{2}}\right)_{p p}
\end{array}\right\} .
$$


The symbol ()$_{p p}$ in this expression represents Cauchy's principle part that results from taking the limit: $\varphi \rightarrow 0$. This frequency shift is the signature of the formation of hybridization band gaps between the zeroth-order and the first-order dispersion relations at the resonance wave numbers. Particular solutions of Equation (9) will also include terms in $e^{i\left(\omega_{0}\left(k^{\prime} \circ k^{\prime \prime}\right) \pm 2 \Omega\right) \tau_{0}}$. These terms introduce additional dispersion curves in the band structure of the time-dependent superlattice obtained by shifting the zeroth-order band structure by $\pm 2 \Omega$ (see Fig. 1(c)). The denominators of the resonance conditions $\omega_{0}^{2}\left(k^{\prime}\right)-\left(\omega_{0}(k+g)-\Omega\right)^{2}=0$ and $\omega_{0}^{2}\left(k^{\prime \prime}\right)-\left(\omega_{0}(k+g)+\Omega\right)^{2}=0$ determine the location of the formation the two hybridization gaps observed in Fig. 1(c). These conditions predict hybridization gaps where the lowest first-order dispersion branch $(g=0)$ and second lowest branch $\left(g=\frac{2 \pi}{L}\right)$ intersect a first-order dispersion curve. The two gaps form only on one side (positive or negative side) of the first Brillouin zone depending on the sign of $\Omega$ (i.e., the direction of propagation of the modulation of the stiffness). These two gaps occur at the same wave number: $k_{g}$. This leads to a band structure that does not possess mirror symmetry about the frequency axis as seen in Figure 1(c). The band structure now possesses a center of inversion, the origin, rather than a mirror plane.

\section{Topology of elastic wave functions}

We can shed light on the non-conventional topology of the displacement Bloch function in the time-dependent superlattice by following a closed continuous path in wave number space and monitoring the phase difference acquired by the amplitude of the wave function over the course of such a cycle. ${ }^{22}$ Figure $1(c)$ is used to illustrate this path. We start from the origin of the band structure $(k=0$, point A) and follow the lowest zeroth-order branch $(g=0)$ by moving in the direction of increasing $k$. We approach the first hybridization gap near $+k_{g}$. As we pass through the hybridization gap, the wave function transitions from a state corresponding to a zeroth-order type wave $\left(e^{i \omega_{0}(k+g) \tau_{0}}\right)$ to a wave having the characteristics of a first-order wave $\left(e^{i\left(\omega_{0}\left(k^{\prime}\right)-\Omega\right) \tau_{0}}\right)$. The transition between these two types of solutions corresponds to a geometric phase difference of $\pi$. Once through the hybridization gap, one then reaches the edge of the Brillouin zone $\left(k=\frac{\pi}{L}\right.$, point B). Since the wave functions are Bloch waves, point $B$ is equivalent by translational symmetry to point $\mathrm{B}^{\prime}$ located on the other edge of the Brillouin zone $\left(k=-\frac{\pi}{L}\right)$. From point $\mathrm{B}^{\prime}$, one then follows the first-order branch corresponding to a wave of the form $\left(e^{i\left(\omega_{0}\left(k^{\prime \prime}\right)-\Omega\right) \tau_{0}}\right)$ back to the wavenumber $k=0$ (point $\mathrm{C}$ ). At this stage, we have closed a $\frac{2 \pi}{L}$ loop in wave number space, and the amplitude of the wave function has accumulated a geometric phase of $\pi$. Further increase in wave number takes us back along the first-order branch corresponding to the wave: $e^{i\left(\omega_{0}\left(k^{\prime}\right)-\Omega\right) \tau_{0}}$. One then reaches the top of the hybridization gap, again at $+k_{g}$, and transitions back to the zerothorder state $e^{i \omega_{0}(k+g) \tau_{0}}$. This transition accumulates an additional geometric phase difference of $\pi$. At the $k=\frac{\pi}{L}$ edge of the Brillouin zone, one has reached the point D. Point $\mathrm{D}$ is equivalent by translational periodicity to $\mathrm{D}^{\prime}$. We can close the continuous path by increasing $k$ again toward the origin along the lowest dispersion branch of the zerothorder wave. This action takes us back to the starting point A. This second stage of our continuous path corresponds to closing a second $\frac{2 \pi}{L}$ loop in $k$-space. For each complete loop, the displacement function accumulated a $\pi$ geometric phase when one crosses the wave number $+k_{g}$, i.e., when transitioning between zeroth-order and first-order wave functions at the gap. One therefore needs to complete two loops in $k$ space (i.e., a $4 \pi / L$ rotation) to obtain a $2 \pi$ geometric phase difference in the amplitude of the wave function. This behavior is characteristic of a non-trivial topology of $k$-space whereby the wave function is supported on a wavenumber manifold that has the torsional topology of a Möbius strip (Fig. 1(e)). ${ }^{23}$ Note that here the twist in the Möbius strip-like manifold is not distributed along the entire length of the strip but is localized in $k$-space. The strip exhibits no phase difference along most of its length. The local twist leads to a $\pi$ phase difference only near $+k_{g}$ which is associated with the narrow gap resulting from fully destructive interferences between first-order and zeroth-order waves. We can represent the evolution of the geometric phase of the wave function by following a closed path in $k$-space on this Möbius strip and parallel transporting a tangent vector field. Starting at point $\mathrm{A}$ in Figure 1(e), and following a closed loop in $k$ space, the upward-pointing tangent vector remains parallel until it approached the twist in the strip at $+k_{g}$. The parallel transport condition imposed on the vector leads to a $\pi$-inversion of the direction in which the vector points. The vectors remain parallel to each other through a full loop ( $\frac{2 \pi}{L}$ rotation) in $k$-space reaching point $\mathrm{C}$. One needs another full turn to go through the twist a second time and rotate the vector by $\pi$ again. The vectors remain parallel until they close the continuous path and reach the point $\mathrm{A}$. The vector has accumulated a $2 \pi$ phase different along a $\frac{4 \pi}{L}$ closed path. In contrast, we have illustrated in Figure 1(d) the manifold for a Bloch wave with conventional topology that corresponds to a timeindependent superlattice. In this case, the manifold does not possess a twist. The vector field is transported along a single close path in $k$-space without a change in phase. The amplitude of the wave function in this case does not depend on the wave number.

In the following sections, through a series of numerical simulations, we demonstrate the application of the concept of time-dependent modulation of elastic properties by showing bulk wave propagation functionalities such as nonreciprocal transmission and immunity to back-scattering.

\section{Non-reciprocity of bulk elastic wave propagation}

Hybridization between zeroth-order and first-order wave functions is permissible only if they possess the same symmetry. As shown with multiple time scales perturbation theory, the intersection of zeroth-order and first-order dispersion branches may or may not result in the formation of a hybridization gap. When the displacement fields associated with the Eigenmodes of each branch are symmetrical, as was the case at $+k_{g}$ in Figure 1(c), a hybridization gap emerges. If symmetry is absent, zeroth-order and first-order dispersion 


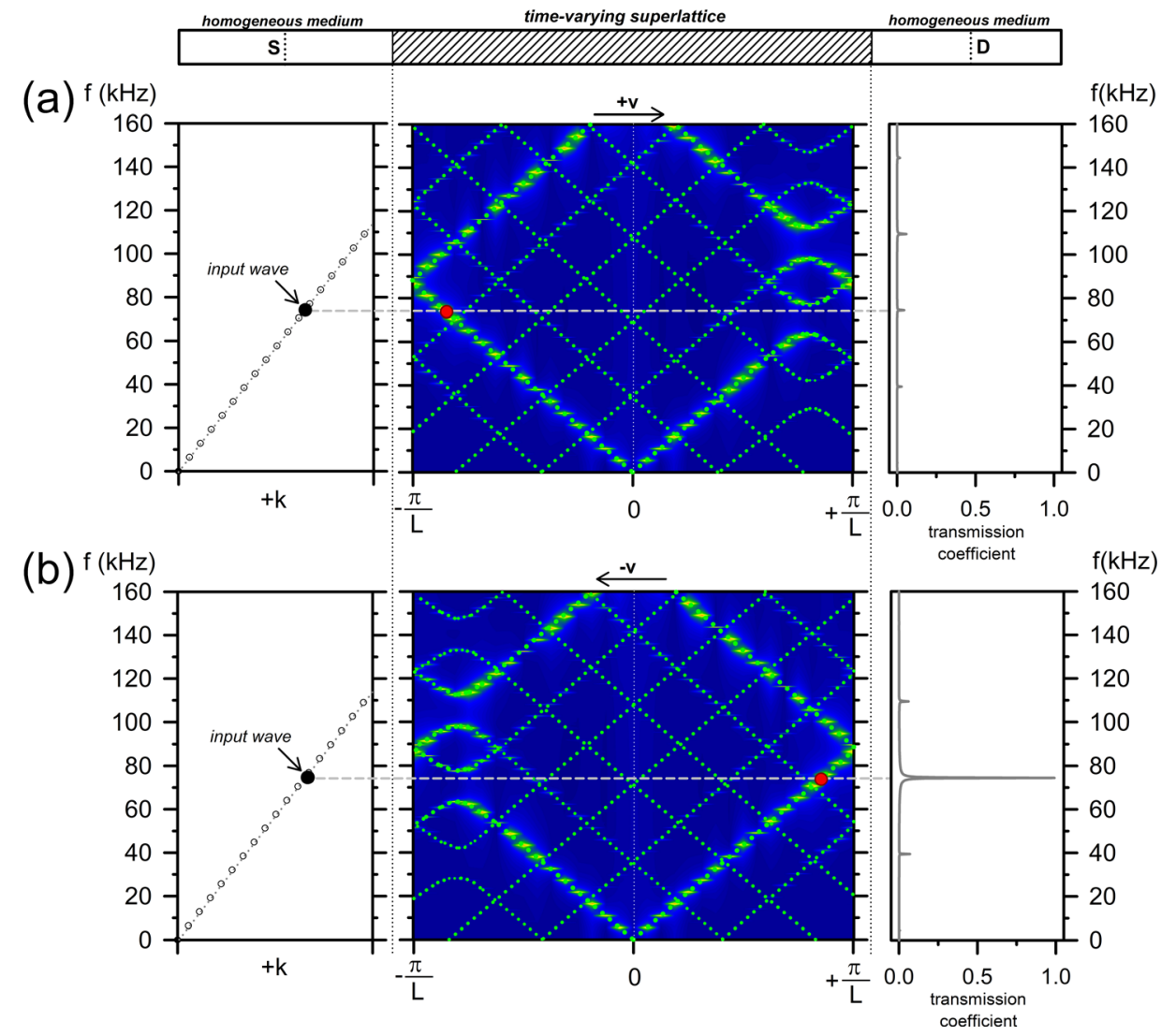

FIG. 2. One-way transport of bulk elastic waves: Illustration of the condition for transmission and transmission coefficient of a finite size timedependent superlattice sandwiched between two homogeneous regions (inlet with sound source $(S)$ and outlet with detector $(D)$ in the case (a) $\mathrm{V}>0$ and (b) $\mathrm{V}<0$. In both cases, the band structure of the homogeneous medium is represented on the left of the figure with the source emitting a monochromatic wave with frequency $f_{0}$. The plots on the right represent the transmission spectrum of the superlattice around the incident frequency.

branches can intersect and not hybridize. The loss of mirror symmetry in the band structure leads to the existence of unidirectional bulk propagative modes within the frequency range of the hybridization gap. This asymmetry can then be exploited to achieve non-reciprocal wave propagation.

To illustrate this phenomenon, we consider a finite system composed of a time-dependent superlattice sandwiched between two homogeneous domains (see top of Figure 2). This setup is particularly useful for simulating the transmission of elastic waves through phononic systems. Absorbing boundary conditions are imposed at the ends of the sandwich-system. The medium to the left of the superlattice contains a source $(S)$ of monochromatic elastic waves. A detector $(D)$ is located in the medium to the right of the superlattice. The spectral properties of the superlattice are characterized by taking the Fourier transform of the timedomain signal collected at $D$. The sandwich-system is discretized and transmission is investigated numerically by solving the elastic wave equation via the finite-difference time-domain (FDTD) methodology. ${ }^{24}$

We consider two simulations, Case I and Case II. For Case I, the frequency of $S$ is set at $f_{0}=72 \mathrm{kHz}$ and the spatial modulation of stiffness propagates in time with velocity $\mathrm{V}>0$. Case II is identical to Case I except the sign of the velocity is switched $(\mathrm{V}<0)$. The dispersion diagrams for the superlattices considered in Case I and Case II are shown in the centers of Figures 2(a) and 2(b), respectively. As noted previously, changing the sign of the modulation velocity leads to a horizontal flip of the band structure. To the left of each diagram is the band structure for acoustic waves in the homogeneous domain. To the right of each diagram is the plot for acoustic wave transmission. It is valuable to use the left and central plots in Figures 2(a) and 2(b) as slowness surfaces to interpret the transmission plots for Case I and Case II. A horizontal grey line is drawn over the band structures of the superlattices considered. This line coincides with the frequency of the input acoustic source. All zeroth-order modes in the superlattices with this frequency will be excited. A red dot is used to identify these modes in Figures 2(a) and 2(b). For Figure 2(a), this mode has negative group velocity and does not propagate in the same direction as the input acoustic wave. As a consequence, the transmission peak at $f_{0}$ is very weak. Oppositely, the red dot in Figure 2(b) highlights a zeroth-order mode with positive group velocity and a very large transmission peak is witnessed at $f_{0}$. Furthermore, excitation of the zeroth-order modes at the frequency $f_{0}$ leads through Equation (7) to first-order modes at the frequencies $f_{0} \pm \Omega / 2 \pi$ (see Equation (8)). These modes appear as small peaks in Figures 2(a) and 2(b).

The unidirectional propagation of elastic waves in the time-dependent superlattice is enabled by the asymmetric band structure and therefore the existence of a bulk zerothorder propagative mode within the frequency range of a band gap. The change in sign of the group velocity of the propagative mode with the sign of the stiffness modulation velocity leads to the asymmetry in transmission coefficient. We note that the characteristics of the transmission coefficient are inverted when one launches an incident wave with a frequency, $f_{1}=105 \mathrm{kHz}$, falling within a different hybridization gap. This inversion results from the change in sign of the group velocity of the propagative mode associated with band folding. 


\section{Demonstration of topologically back-scattering-immune bulk states}

One of the signatures of edge states in topological insulators is their robustness with respect to backscattering by defects. The same robustness exists for the bulk elastic waves in the time-dependent superlattice. This phenomenon is demonstrated by inserting a mass defect inside the elastic sandwich-system considered previously. Here, the defect is constructed by changing the value of the masses of a region of the superlattice (see Fig. 3(a)). We consider increasing levels of mismatch between the defect and the original superlattice. The defect mass is chosen to take the values $M=2$, 3 , and $4 m$. The width of this region is taken to be equal to the period of the modulation. The mass defect does not affect the spatio-temporal modulation of the stiffness.

The incident wave has the same frequency $f_{0}$ from Fig. 2, whereby unidirectional propagation is attained. As can be seen in Fig. 3(b), the transmission of the bulk wave through the time-independent superlattice is increasingly degraded by an increase in the mass mismatch, i.e., backscattering. However, the time-dependent superlattice exhibits no significant transmission for $\mathrm{V}<0$ as expected. For $\mathrm{V}>0$, the mass

(a)

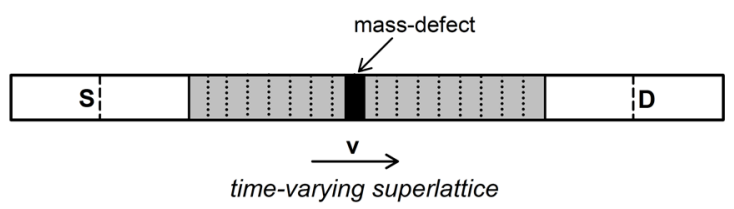

(b)

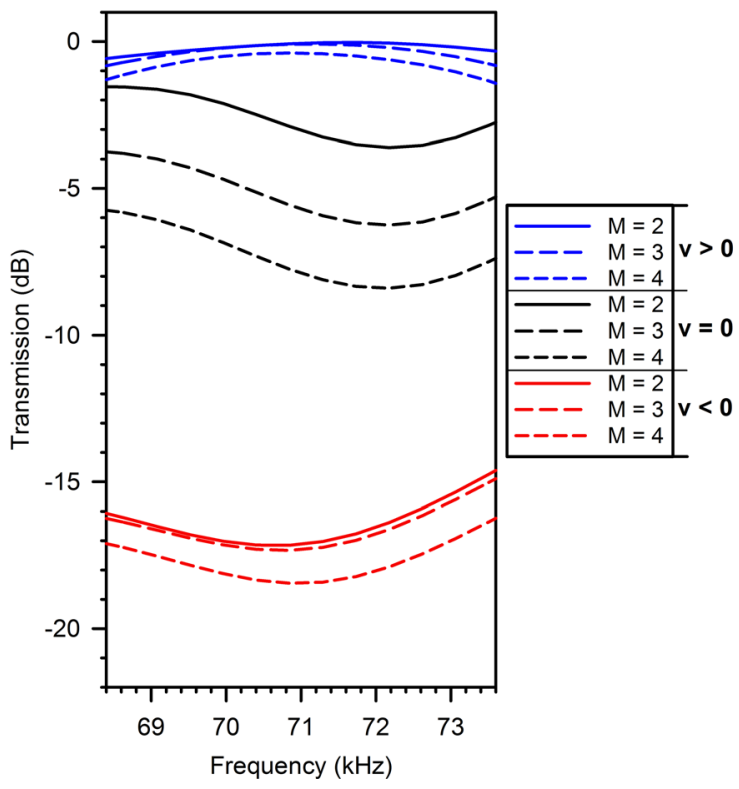

FIG. 3. Immunity to backscattering of bulk elastic waves. (a) Schematic representation of the sandwich system of Figure 2 with a mass defect (see text for details). (b) Transmission through the defected superlattice. The incident wave has a frequency $f_{\mathrm{o}}=72 \mathrm{kHz}$ for which propagation is topologically constrained to be unidirectional. The blue and red curves correspond to time-dependent superlattices with opposite modulation velocities. The black curves represent transmission though a time-independent superlattice. Solid, short-dashed, and long-dashed curves correspond to increasing levels of mass mismatch between the defect and the superlattice. of the defect does not appear to have a significant effect on the transmission. Since the superlattice is unable in this case of supporting a defect induced back-scattered wave, the elastic energy essentially propagates without scattering. The time-dependent superlattice demonstrates unambiguously immunity to backscattering.

\section{SUMMARY AND OUTLOOK}

We have identified the non-conventional topology of bulk elastic waves in a time-dependent superlattice as well as demonstrated the existence of bulk elastic waves with unidirectional backscattering-immune topological states. The moving spatial modulation of the elastic constants leads to modes with split frequency. The splitting is linear with respect to the biasing velocity. It is the band folding due to the spatial modulation which enables hybridization between the split Bloch modes and the Bloch modes of the timeindependent superlattice. The hybridization opens gaps in a band structure that has lost its mirror symmetry about the origin of the Brillouin zone. The elastic wave function is supported in wave-number space by a Möbius strip-like manifold with non-conventional torsional topology. These topology protected bulk states exhibit unidirectional propagation and immunity to back scattering by defects.

The mechanism of asymmetric hybridization between Bloch waves and frequency-split Bloch waves in a timedependent superlattice, demonstrated here for elastic waves, is universal. We anticipate that this concept will develop into approaches to realize topology protected bulk states in materials supporting other types of waves such as electromagnetic waves or spin waves. Although we have illustrated the concept of the spatio-temporal modulation of the elastic properties in a photo-elastic medium, one can achieve time-dependent superlattices in a variety of other classes of materials by exploiting, for instance, the magneto-elastic effect or the piezo-electric effect. We have considered a one-dimensional sinusoidal spatio-temporal modulation of the properties of the material. The universality of the concept reported here suggests its extension to materials with higher dimensions and other more complex forms of the spatio-temporal modulation. The concept of time-dependent materials that can break time reversal symmetry for bulk wave propagation may potentially serve as unique platforms to investigate a large variety of phenomena resulting from wave propagation with non-conventional topological states.

${ }^{1}$ M. Z. Hasan and C. L. Kane, Rev. Mod. Phys. 82, 3045-3067 (2010).

${ }^{2}$ A. B. Khanikaev, S. H. Mousavi, W.-K. Tse, M. Kargarian, A. H. MacDonald, and G. Shvets, Nat. Mater. 12, 233-239 (2013).

${ }^{3}$ M. C. Rechtsman, J. M. Zeuner, Y. Plotnik, Y. Lumer, D. Podolsky, F. Dreisow, S. Nolte, M. Sergev, and A. Szameit, Nature 496, 196-200 (2013).

${ }^{4}$ F. D. M. Haldane and S. Raghu, Phys. Rev. Lett. 100, 013904 (2008).

${ }^{5}$ F. Li, P. Anzel, J. Yang, P. G. Kevrekidis, and C. Daraio, Nat. Commun. 5, 5311 (2014).

${ }^{6}$ B.-I. Popa and S. A. Cummer, Nat. Commun. 5, 3398 (2014).

${ }^{7}$ B. W. Li, L. Wang, and G. Casati, Phys. Rev. Lett. 93, 184301 (2004).

${ }^{8}$ B. Liang, X. S. Guo, J. Tu, D. Zhang, and J. C. Cheng, Nat. Mater. 9, 989-992 (2010). 
${ }^{9}$ C. Kittel, Phys. Rev. 110, 836-841 (1958).

${ }^{10}$ R. Fleury, D. L. Sounas, C. F. Sieck, M. R. Haberman, and A. Alu, Science 343, 516-519 (2014).

${ }^{11}$ V. Yannopapas, New J. Phys. 14, 113017 (2012).

${ }^{12}$ V. Yannopapas, J. Opt. 14, 085105 (2012).

${ }^{13}$ Z. Yu and S. Fan, Nat. Photonics 3, 91-94 (2009).

${ }^{14}$ D.-W. Wang, H.-T. Zhou, M.-J. Guo, J.-X. Zhang, J. Evers, and S.-Y. Zhu, Phys. Rev. Lett. 110, 093901 (2013).

${ }^{15}$ J. N. Winn, S. Fan, E. P. Ippen, and J. D. Joannopoulos, Phys. Rev. B 59, 1551-1554 (1999).

${ }^{16}$ D. W. Wright and R. S. C. Cobbold, Smart Mater. Struct. 18, 015008 (2009).
${ }^{17}$ F. Li, C. Chong, J. Yang, P. G. Kevrekidis, and C. Daraio, Phys. Rev. E 90, 053201 (2014).

${ }^{18}$ M. B. Zanjani, A. R. Davoyan, A. M. Mahmoud, N. Engheta, and J. R. Lukes, Appl. Phys. Lett. 104, 081905 (2014).

${ }^{19}$ J. Gump, I. Finckler, H. Xia, R. Sooryakumar, W. J. Bresser, and P. Boolchand, Phys. Rev. Lett. 92, 245501 (2004).

${ }^{20}$ J. A. Thomas, J. E. Turney, R. M. Iutzi, C. H. Amon, and A. J. H. McGaughey, Phys. Rev. B 81, 081411 (2010).

${ }^{21}$ R. H. G. Helleman and E. W. Montroll, Physica 74, 22-74 (1974).

${ }^{22}$ M. V. Berry, Proc. R. Soc. A 392, 45-57 (1984).

${ }^{23}$ H. C. Manoharan, Nat. Nanotechol. 5, 477-479 (2010).

${ }^{24}$ Y. Tanaka, Y. Tomoyasu, and S. Tamura, Phys. Rev. B 62, 7387 (2000). 